Supporting Information for

\title{
Wafer-scale Nanopillars Derived from Block Copolymer Lithography for Surface-enhanced Raman Spectroscopy
}

Tao Li*, Kaiyu Wu*, Tomas Rindzevicius, Zhongli Wang, Lars Schulte, Michael S. Schmidt, Anja Boisen and Sokol Ndoni

Department of Micro- and Nanotechnology

Technical University of Denmark,

Anker Engelunds Vej 1, Kgs. Lyngby, 2800, Denmark.

E-mail: taoli@,nanotech.dtu.dk ; kaiwu@nanotech.dtu.dk

This PDF file includes:

Experimental setup for solvent vapor annealing

Optical images of SD172 thin film on silicon wafer after solvent annealing

A top-view SEM image of the silicon nanohole array after ALD

An optical image of the gold nanopillar substrate near a droplet of analyte solution 


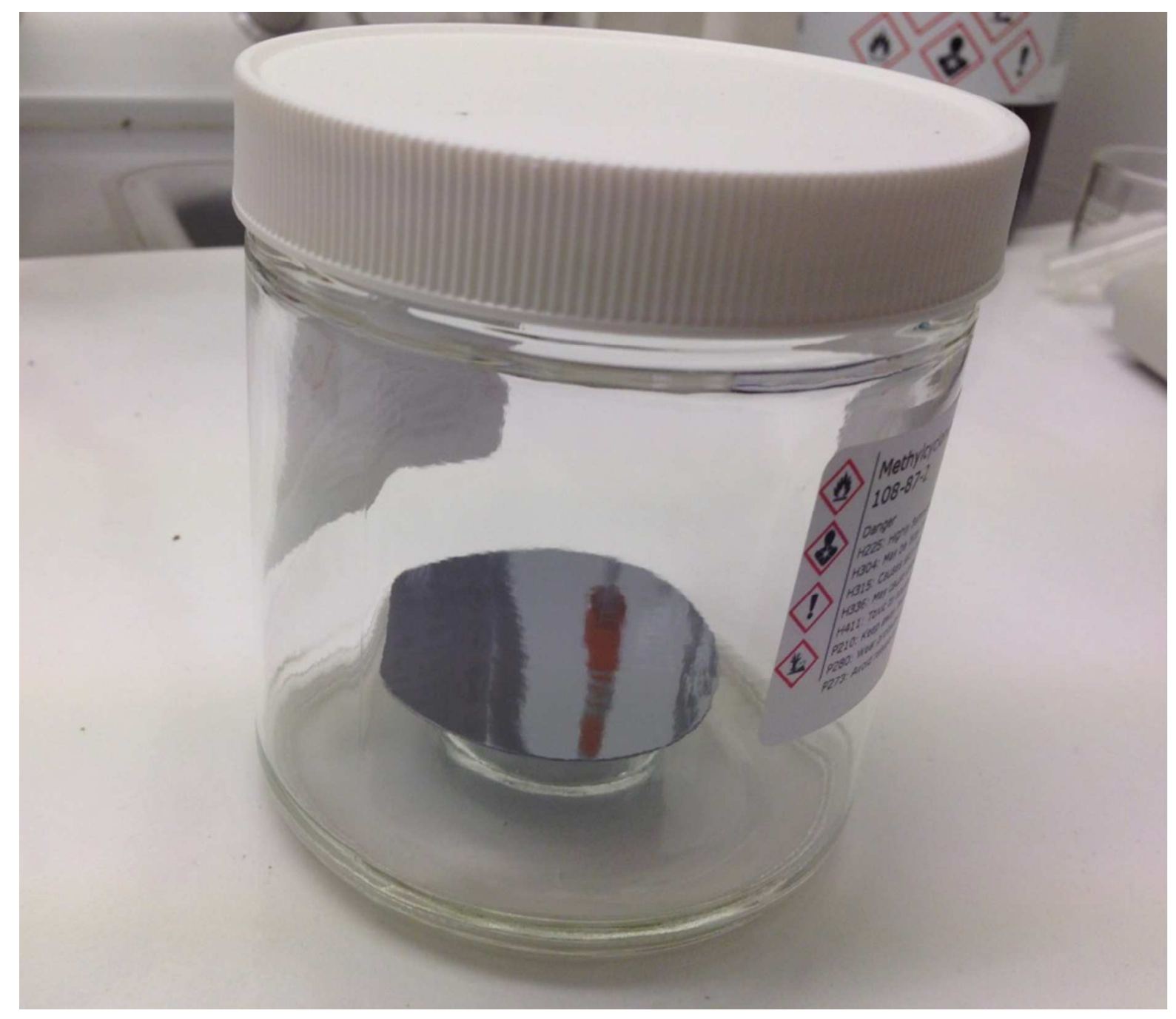

Figure S1. Experimental setup for solvent vapor annealing. 


\section{a}
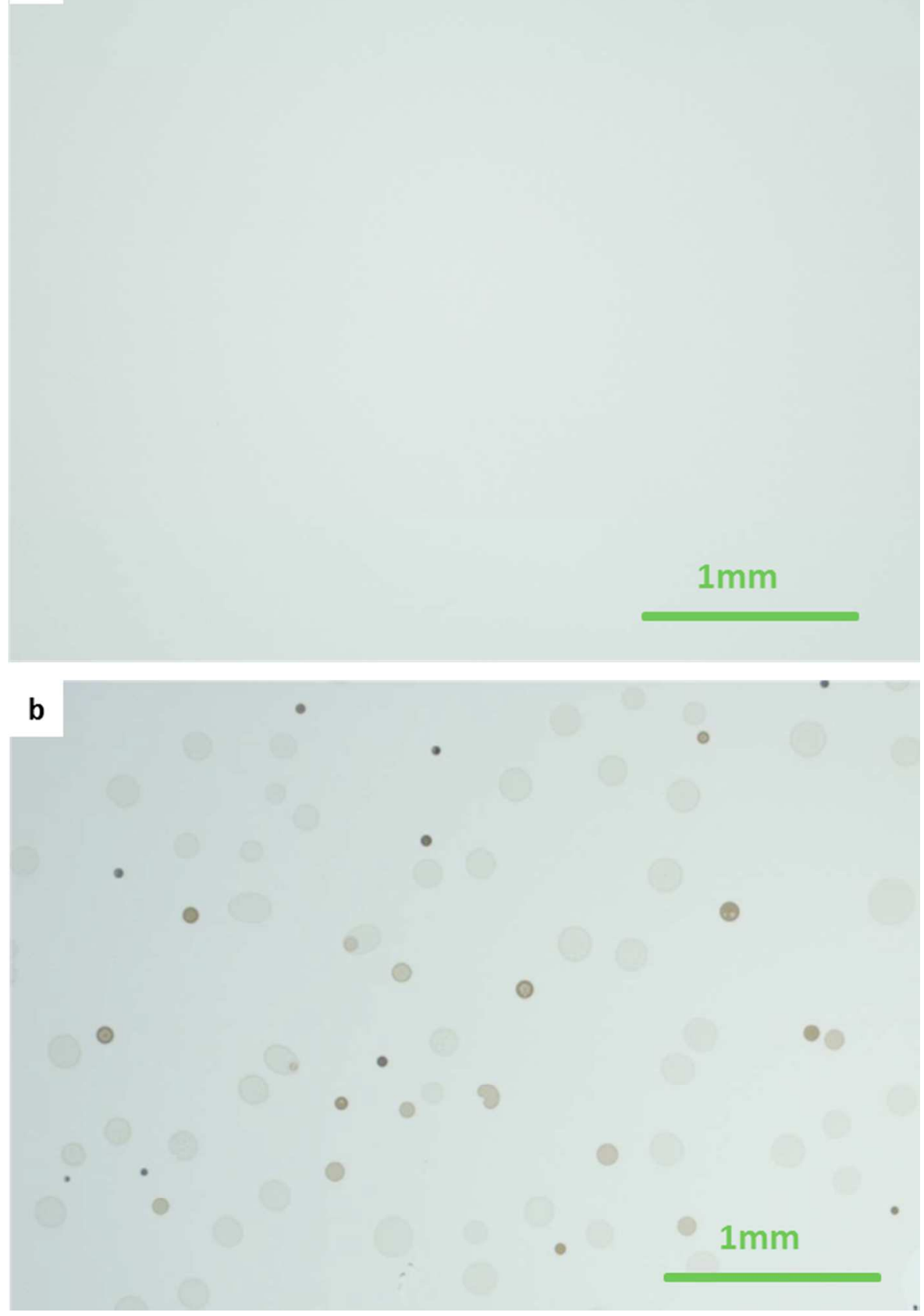

Figure S2. (a) An optical image of SD172 thin film on silicon wafer after solvent annealing, showing a random area over $4 \mathrm{~mm}$ by $3 \mathrm{~mm}$, which is seen to be defect-free. (b) In contrast, a film annealed in toluene for 20 minutes shows de-wetting/terrace domains, which are clearly visible in an optical microscope image. 


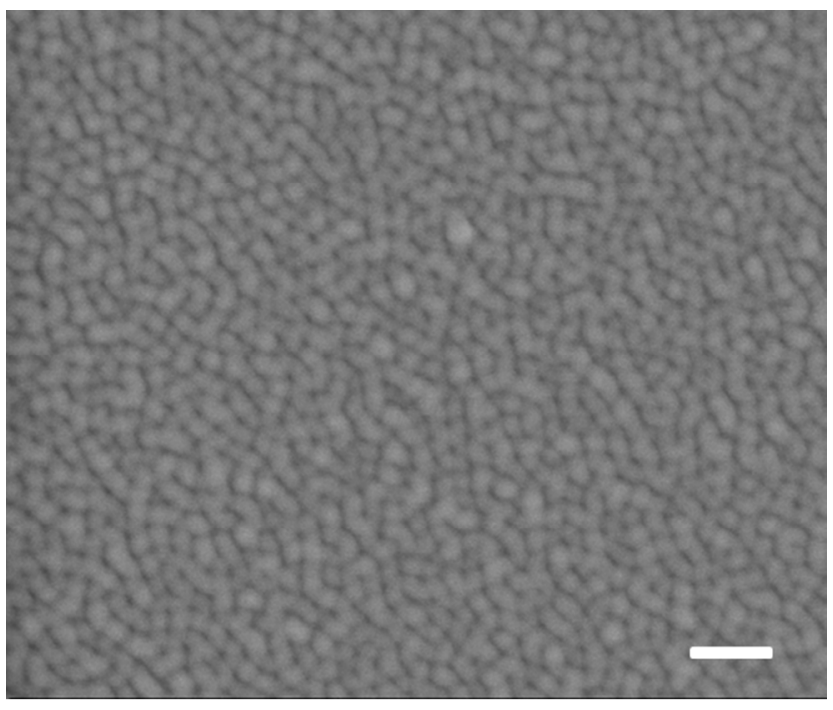

Figure S3. A top-view SEM image of the silicon nanohole array fabricated by SD172 after $\mathrm{Al}_{2} \mathrm{O}_{3}$ ALD. Scale bar: $200 \mathrm{~nm}$. 


\section{$3 \mathrm{~mm}$}

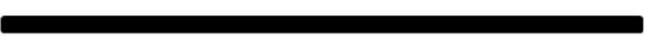

Figure S4. An optical image of the gold nanopillar substrate near a droplet of analyte solution 